\title{
Pyranocycloartobiloxanthone A, a novel gastroprotective compound from Artocarpus obtusus Jarret, against ethanol-induced acute gastric ulcer in vivo
}

\begin{abstract}
Pyranocycloartobiloxanthone A (PA), a xanthone derived from the Artocarpus obtusus Jarret, belongs to the Moraceae family which is native to the tropical forest of Malaysia. In this study, the efficacy of PA as a gastroprotective compound was examined against ethanolinduced ulcer model in rats. The rats were pretreated with PA and subsequently exposed to acute gastric lesions induced by absolute ethanol. The ulcer index, gastric juice acidity, mucus content, histological analysis, glutathione (GSH) levels, malondialdehyde level (MDA), nitric oxide (NO) and non-protein sulfhydryl group (NP-SH) contents were evaluated in vivo. The activities of PA as anti-Helicobacter pylori, cyclooxygenase-2 (COX-2) inhibitor and free radical scavenger were also investigated in vitro. The results showed that the oral administration of PA protects gastric mucosa from ethanol-induced gastric lesions. PA pretreatment significantly $(\mathrm{p}<0.05)$ restored the depleted GSH, NP-SH and NO levels in the gastric homogenate. Moreover, PA significantly $(\mathrm{p}<0.05)$ reduced the elevated MDA level due to ethanol administration. The gastroprotective effect of PA was associated with an over expression of HSP70 and suppression of Bax proteins in the ulcerated tissue. In addition, PA exhibited a potent FRAP value and significant COX-2 inhibition. It also showed a significant minimum inhibitory concentration (MIC) against $H$. pylori bacterium. The efficacy of PA was accomplished safely without the presence of any toxicological parameters. The results of the present study indicate that the gastroprotective effect of PA might contribute to the antioxidant and anti-inflammatory properties as well as the anti-apoptotic mechanism and antibacterial action against Helicobacter pylori.
\end{abstract}

Keyword: Artocarpus obtusus; Pyranocycloartobiloxanthone A; Gastroprotection; HSP70; Antioxidant; Helicobacter pylori. 